\title{
Acrylamide in the Baylis-Hillman Reaction: Expanded \\ Reaction Scope and the Unexpected Superiority of \\ DABCO Over More Basic Tertiary Amine Catalysts
}

Cornelia Faltin, Eimear M. Fleming and Stephen J. Connon*

Department of Chemistry, Trinity College Dublin, Dublin 2, Ireland.

connons@tcd.ie 
$\begin{array}{llr}1.0 & \text { General } & \text { S3 }\end{array}$

$\begin{array}{lll}2.0 & \text { Experimental details } & \text { S3 }\end{array}$

2.1 Kinetic studies on the hydroalkoxylation reaction (Table 1) S3

2.2 Baylis-Hillman reaction involving acrylamide and various aldehydes S4

$\begin{array}{lll}\text { 2.3 Characterisation data for Baylis-Hillman adducts } & \text { S5 }\end{array}$

2-[Hydroxy(2-nitrophenyl)methyl]acrylamide (13) S5

2-[Hydroxy(4-chlorophenyl)methyl]acrylamide (14) S5

2-[Hydroxy(phenyl)methyl]acrylamide (4)

S6

2-[Hydroxy(2-naphthyl)methyl]acrylamide (15) S6

2-[Hydroxy(4-methylphenyl)methyl]acrylamide (16) S6

2-[Hydroxy(2-methylphenyl)methyl]acrylamide (17) $\quad$ S7

2-[Hydroxy(2-methoxyphenyl)methyl]acrylamide (6) S7

$\begin{array}{lll}\text { 3.0 } & { }^{1} \text { H NMR Spectra } & \text { S8 }\end{array}$

2-[Hydroxy(2-nitrophenyl)methyl]acrylamide (13) S8

2-[Hydroxy(4-chlorophenyl)methyl]acrylamide (14) S8

2-[Hydroxy(phenyl)methyl]acrylamide (4)

S9

2-[Hydroxy(2-naphthyl)methyl]acrylamide (15) S9

2-[Hydroxy(4-methylphenyl)methyl]acrylamide (16) S10

2-[Hydroxy(2-methylphenyl)methyl]acrylamide (17) S10

2-[Hydroxy(2-methoxyphenyl)methyl]acrylamide (6) S11

$\begin{array}{ll}\text { Hydromethoxylation product (3a) } & \text { S11 }\end{array}$

Hydromethoxylation product (3a) $-{ }^{13} \mathrm{C}$ NMR and DEPT 135 spectra S12 


\subsection{References}

\subsection{General}

Proton Nuclear Magnetic Resonance spectra were recorded on a $400 \mathrm{MHz}$ spectrometer in $\mathrm{CDCl}_{3}$ referenced relative to residual $\mathrm{CHCl}_{3}(\delta=7.26 \mathrm{ppm})$. Chemical shifts are reported in ppm and coupling constants in Hertz. Carbon NMR spectra were recorded on the same instrument $(100 \mathrm{MHz})$ with total proton decoupling. All melting points are uncorrected. Flash chromatography was carried out using silica gel, particle size $0.04-0.063 \mathrm{~mm}$. TLC analysis was performed on precoated $60 \mathrm{~F}_{254}$ slides, and visualised by either UV irradiation or $\mathrm{KMnO}_{4}$ staining. Acrylamide, 4-dimethylaminopyridine (DMAP), 1,8-diazabicyclo[5.4.0] undec-7-ene (DBU), quinuclidine, tri- $n$-butylphosphine, triethylamine (TEA), diisopropylethylamine (DIPEA) and 1,4-diazabicyclo[2.2.2]octane (DABCO) were obtained from commercial sources and used as received. DABCO could be recrystallised/sublimed before use, however this had no observable beneficial effect on reaction rates/yields. All liquid aldehydes, anisole and reaction solvents were distilled before use. $o$-Nitrobenzaldehyde was recrystallised from hexane prior to use.

\section{Experimental Details}

\subsection{Kinetic studies on the hydroalkoxylation reaction (Table 1)}

A $1 \mathrm{~mL}$ vial equipped with a septum and a small stirring bar under Ar (balloon) was charged with a solution of the amine catalyst $(1.0 \mathrm{mmol})$ and acrylamide $(1.0 \mathrm{mmol})$ in $\mathrm{MeOH}(500 \mu \mathrm{L})$. To this was added anisole $(27 \mu \mathrm{L}, 0.25 \mathrm{mmol})$ via syringe (as an internal standard). Samples were taken periodically and analysed by ${ }^{1} \mathrm{H}$ NMR spectroscopy. Two sample pseudo $1^{\text {st }}$ order rate plots are shown below for the hydromethoxylation reaction promoted by quinuclidine. The results are averaged to give the value shown in Table 1 (entry 4). 

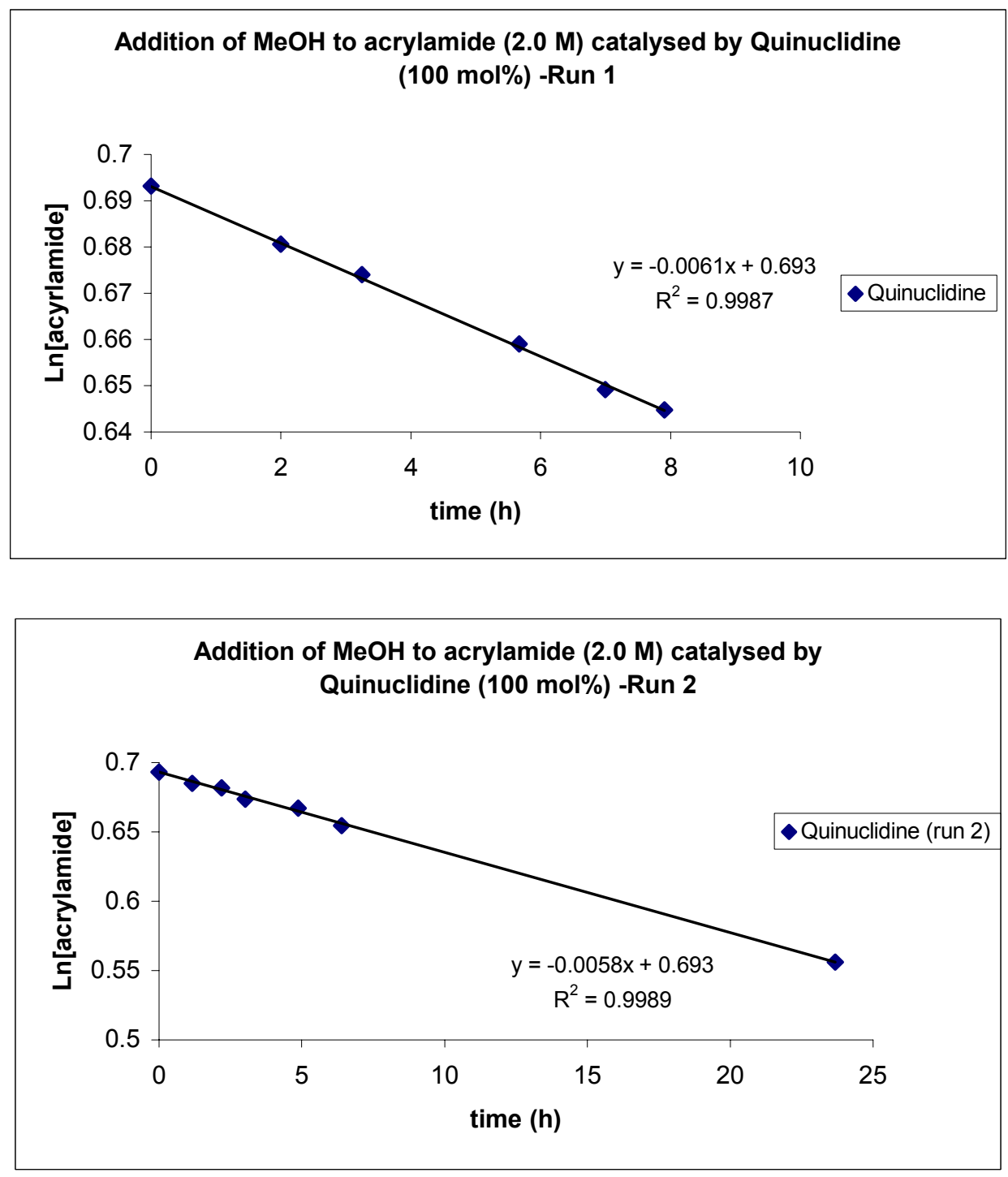

Note: For hydroalkoxylation reactions involving $\mathrm{Bu}_{3} \mathrm{P}$, the methanolic solvent was subjected to three freeze-thaw vacuum cycles before reaction.

\subsection{Baylis-Hillman reaction involving acrylamide and various aldehydes}

Procedure using condition set A: A $1 \mathrm{~mL}$ reaction vessel equipped with a stirring bar was charged with acrylamide (142 mg, $2.0 \mathrm{mmol})$, DABCO (224 mg, $2.0 \mathrm{mmol})$ and phenol (47 mg, $0.5 \mathrm{mmol})$. Note: If the aldehyde component is a solid it is added at this stage. The vessel was fitted with a septum, gently flushed with Ar gas and fitted with an Ar balloon. A ${ }^{t} \mathrm{BuOH} / \mathrm{H}_{2} \mathrm{O}$ solvent mixture $(120 \mu \mathrm{L})$ and the aldehyde $(3.0 \mathrm{mmol})$ were then added via syringe. The resulting homogeneous mixture was stirred at $55{ }^{\circ} \mathrm{C}$ for the time indicated in Table 3 and allowed to cool. Column chromatography of the reaction mixture $\left(95: 5 \mathrm{CH}_{2} \mathrm{Cl}_{2} / \mathrm{MeOH}\right)$ yielded the required Baylis-Hillman adducts. 
Procedure using condition set B: A $1 \mathrm{~mL}$ reaction vessel equipped with a stirring bar was charged with acrylamide (142 mg, $2.0 \mathrm{mmol})$, DABCO (224 mg, $2.0 \mathrm{mmol})$ and phenol (188 mg, $2.0 \mathrm{mmol})$. Note: If the aldehyde component is a solid it is added at this stage. The vessel was fitted with a septum, gently flushed with Ar gas and fitted with an Ar balloon. The aldehyde $(3.0 \mathrm{mmol})$ was then added via syringe. The resulting mixture was heated to $55^{\circ} \mathrm{C}$ (whereupon it became homogeneous) and stirred at this temperature for the time indicated in Table 3 and then allowed to cool. Column chromatography of the reaction mixtures $\left(95: 5 \mathrm{CH}_{2} \mathrm{Cl}_{2} / \mathrm{MeOH}\right)$ yielded the required Baylis-Hillman adducts.

\subsection{Characterisation data for Baylis-Hillman adducts ${ }^{1}$}

\section{2-[Hydroxy(2-nitrophenyl)methyl]acrylamide (13)}

Spectral data consistent with that reported in the literature ${ }^{2}$

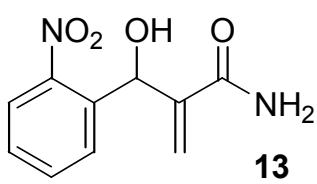

M.p. $136-138{ }^{\circ} \mathrm{C}$; IR (film) 3446, 3215, 2925, 1666, $1523 \mathrm{~cm}^{-1} ;{ }^{1} \mathrm{H}$ NMR (400 MHz, DMSO- $\left.d_{6}\right) \delta 5.48$

(s, 1H), $5.89(\mathrm{~s}, 1 \mathrm{H}), 5.94(\mathrm{~d}, 1 \mathrm{H}, \mathrm{J}=5.5 \mathrm{~Hz}), 6.05(\mathrm{~d}, 1 \mathrm{H}, \mathrm{J}=5.5 \mathrm{~Hz}), 7.02(\mathrm{~s}, 1 \mathrm{H}), 7.48(\mathrm{t}, 1 \mathrm{H}, \mathrm{J}=7.5$

$\mathrm{Hz}), 7.57(\mathrm{~m}, 2 \mathrm{H}), 7.65(\mathrm{t}, 1 \mathrm{H}, \mathrm{J}=7.5 \mathrm{~Hz}), 7.84(\mathrm{~d}, 1 \mathrm{H}, \mathrm{J}=8.0 \mathrm{~Hz}) ;{ }^{13} \mathrm{C}$ NMR $\left(100 \mathrm{MHz}, \mathrm{DMSO}-d_{6}\right) \delta$

65.7, 118.3, 123.8, 128.3, 128.9, 132.8, 137.3, 146.2, 148.5, 168.1; HRMS (EI) $m / z\left(\mathrm{MH}^{+}\right)$calcd for $\mathrm{C}_{10} \mathrm{H}_{11} \mathrm{~N}_{2} \mathrm{O}_{4}$ 223.0719, found 223.0712; Anal. Calcd for $\mathrm{C}_{10} \mathrm{H}_{10} \mathrm{~N}_{2} \mathrm{O}_{4} \mathrm{C}, 54.05 ; \mathrm{H}, 4.54 ; \mathrm{N}, 12.61$. Found C, 53.85; H, 4.50; N, 12.37 .

\section{2-[Hydroxy(4-chlorophenyl)methyl]acrylamide (14)}

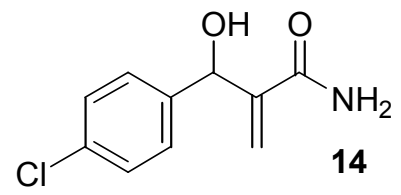

M.p. $132-134{ }^{\circ} \mathrm{C}$; IR (film) 3338, 3203, 2885, 1662, 1624, 1596, $1490 \mathrm{~cm}^{-1}$; ${ }^{1} \mathrm{H}$ NMR (400 MHz, DMSO-d $)_{6} \delta 5.49(\mathrm{~d}, 1 \mathrm{H}, \mathrm{J}=4.5 \mathrm{~Hz}), 5.62(\mathrm{~s}, 1 \mathrm{H}), 5.78(\mathrm{~d}, 1 \mathrm{H}, \mathrm{J}=4.5 \mathrm{~Hz}), 5.81(\mathrm{~s}, 1 \mathrm{H}), 7.01(\mathrm{~s}, 1 \mathrm{H})$, $7.30(\mathrm{~d}, 2 \mathrm{H}, \mathrm{J}=8.6 \mathrm{~Hz}), 7.35(\mathrm{~d}, 2 \mathrm{H}, \mathrm{J}=8.6 \mathrm{~Hz}), 7.47(\mathrm{~s}, 1 \mathrm{H}) ;{ }^{13} \mathrm{C}$ NMR $\left(100 \mathrm{MHz}, \mathrm{DMSO}-d_{6}\right) \delta 70.3$, 117.6, 127.9, 128.6, 131.4, 142.4, 147.1, 168.5; HRMS (EI) m/z calcd for $\mathrm{C}_{10} \mathrm{H}_{10} \mathrm{ClNO}_{2} 211.0400$, 
found 211.0412; Anal. Calcd for $\mathrm{C}_{10} \mathrm{H}_{10} \mathrm{ClNO}_{2} \mathrm{C}, 56.75 ; \mathrm{H}, 4.76 ; \mathrm{N}, 6.62$. Found C, 56.48; H, 4.72; N, 6.45 .

\section{2-[Hydroxy(phenyl)methyl]acrylamide (4)}

Spectral data consistent with that reported in the literature ${ }^{3}$

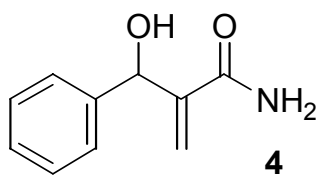

M.p. $97-99{ }^{\circ} \mathrm{C}$; IR (film) 3331, 3212, 3031, 1665, 1626, $1594 \mathrm{~cm}^{-1} ;{ }^{1} \mathrm{H}$ NMR (400 MHz, DMSO-d $) \delta$ $5.49(\mathrm{~d}, 1 \mathrm{H}, \mathrm{J}=4.5 \mathrm{~Hz}), 5.60(\mathrm{~s}, 1 \mathrm{H}), 5.69(\mathrm{~d}, 1 \mathrm{H}, \mathrm{J}=4.5 \mathrm{~Hz}), 5.79(\mathrm{~s}, 1 \mathrm{H}), 6.98(\mathrm{~s}, 1 \mathrm{H}), 7.22(\mathrm{~m}, 1 \mathrm{H})$, $7.30(\mathrm{~m}, 4 \mathrm{H}), 7.45(\mathrm{~s}, 1 \mathrm{H}) ;{ }^{13} \mathrm{C}$ NMR $\left(100 \mathrm{MHz}, \mathrm{DMSO}-d_{6}\right) \delta$ 71.1, 117.4, 126.7, 126.9, 127.9, 143.3, 147.5, 168.7; HRMS (EI) $m / z$ calcd for $\mathrm{C}_{10} \mathrm{H}_{11} \mathrm{NO}_{2}$ 177.0790, found 177.0788; Anal. Calcd for $\mathrm{C}_{10} \mathrm{H}_{11} \mathrm{NO}_{2} \mathrm{C}, 67.78 ; \mathrm{H}, 6.26 ; \mathrm{N}, 7.90$. Found C, 67.29; H, 6.23; N, 7.77.

\section{2-[Hydroxy(2-naphthyl)methyl]acrylamide (15)}

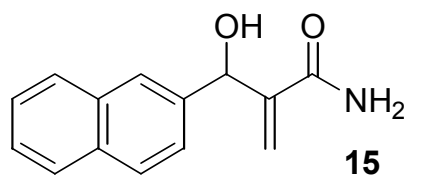

M.p. $132-134{ }^{\circ} \mathrm{C}$; IR (film) 3332, 3212, 3056, 2973, 1665, 1626, $1597 \mathrm{~cm}^{-1}$; ${ }^{1} \mathrm{H}$ NMR (400 MHz, $\left.\mathrm{CDCl}_{3}\right) \delta 3.78(\mathrm{~s}, 1 \mathrm{H}), 5.44(\mathrm{bs}, 1 \mathrm{H}), 5.59(\mathrm{~s}, 1 \mathrm{H}), 5.76(\mathrm{~s}, 1 \mathrm{H}), 6.01(\mathrm{~s}, 1 \mathrm{H}), 6.21(\mathrm{bs}, 1 \mathrm{H}), 7.49(\mathrm{~m}, 3 \mathrm{H})$, $7.84(\mathrm{~m}, 3 \mathrm{H}) 7.91(\mathrm{~s}, 1 \mathrm{H})$; HRMS (EI) $m / z$ calcd for $\mathrm{C}_{14} \mathrm{H}_{13} \mathrm{NO}_{2}$ 227.0946, found 227.0943; Anal. Calcd for $\mathrm{C}_{14} \mathrm{H}_{13} \mathrm{NO}_{2} \mathrm{C}, 73.99 ; \mathrm{H}, 5.77 ; \mathrm{N}, 6.16$. Found $\mathrm{C}, 73.43 ; \mathrm{H}, 5.67 ; \mathrm{N}, 6.12$.

\section{2-[Hydroxy(4-methylphenyl)methyl]acrylamide (16)}

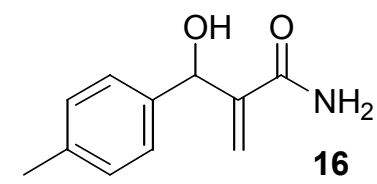

M.p. $113-115^{\circ} \mathrm{C}$; IR (film) 3332, 3212, 2921, 1665, 1627, $1594 \mathrm{~cm}^{-1} ;{ }^{1} \mathrm{H}$ NMR (400 MHz, DMSO-d $) \delta$ $2.27(\mathrm{~s}, 3 \mathrm{H}), 5.45(\mathrm{~d}, 1 \mathrm{H}, \mathrm{J}=5.0 \mathrm{~Hz}), 5.58(\mathrm{~s}, 1 \mathrm{H}), 5.60(\mathrm{~d}, 1 \mathrm{H}, \mathrm{J}=5.0 \mathrm{~Hz}), 5.77(\mathrm{~s}, 1 \mathrm{H}), 6.96(\mathrm{~s}, 1 \mathrm{H})$, $7.09(\mathrm{~d}, 2 \mathrm{H}, \mathrm{J}=7.6 \mathrm{~Hz}), 7.17(\mathrm{~d}, 2 \mathrm{H}, \mathrm{J}=7.6 \mathrm{~Hz}), 7.41(\mathrm{~s}, 1 \mathrm{H}) ;{ }^{13} \mathrm{C}$ NMR $\left(100 \mathrm{MHz}, \mathrm{DMSO}-d_{6}\right) \delta 20.7$, 70.9, 117.1, 126.7, 128.4, 136.0, 140.2, 147.6, 168.1; Anal. Calcd for $\mathrm{C}_{11} \mathrm{H}_{13} \mathrm{NO}_{2} \mathrm{C}, 69.09 ; \mathrm{H}, 6.85 ; \mathrm{N}$, 7.32. Found C, 69.03; H, 6.84; N, 7.58. 
2-[Hydroxy(2-methylphenyl)methyl]acrylamide (17)

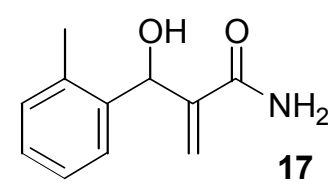

M.p. $133-135^{\circ} \mathrm{C}$; IR (film) $3337,3208,2971,1664,1629,1594 \mathrm{~cm}^{-1} ;{ }^{1} \mathrm{H}$ NMR (400 MHz, DMSO- $\left.d_{6}\right) \delta$ $2.31(\mathrm{~s}, 3 \mathrm{H}) 5.42(\mathrm{~d}, 1 \mathrm{H}, \mathrm{J}=4.0 \mathrm{~Hz}), 5.44(\mathrm{~s}, 1 \mathrm{H}), 5.66(\mathrm{~d}, 1 \mathrm{H}, \mathrm{J}=4.0 \mathrm{~Hz}), 5.78(\mathrm{~s}, 1 \mathrm{H}), 6.97(\mathrm{~s}, 1 \mathrm{H})$, $7.12(\mathrm{~m}, 3 \mathrm{H}), 7.24(\mathrm{~m}, 1 \mathrm{H}), 7.52(\mathrm{~s}, 1 \mathrm{H}) ;{ }^{13} \mathrm{C}$ NMR (100 MHz, DMSO-d 6 ) $\delta$ 23.5, 72.4, 122.0, 130.2, 131.3, 131.7, 134.7, 140.4, 145.7, 152.1, 168.3; HRMS (EI) $\mathrm{m} / z$ calcd for $\mathrm{C}_{11} \mathrm{H}_{13} \mathrm{NO}_{2}$ 191.0946, found 191.0945.

\section{2-[Hydroxy(2-methoxyphenyl)methyl]acrylamide (6)}

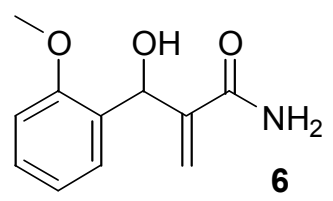

M.p. $164-166{ }^{\circ} \mathrm{C}$; IR (film) 3331, 3209, 2938, 1665, 1628, 1600, $1589 \mathrm{~cm}^{-1} ;{ }^{1} \mathrm{H}$ NMR $(400 \mathrm{MHz}$, DMSO- $\left.d_{6}\right) \delta 3.75(\mathrm{~s}, 3 \mathrm{H}) 5.30(\mathrm{~s}, 1 \mathrm{H}), 5.38(\mathrm{~s}, 1 \mathrm{H}), 5.75(\mathrm{~s}, 1 \mathrm{H}), 5.78(\mathrm{~s}, 1 \mathrm{H}), 6.93(\mathrm{~m}, 3 \mathrm{H}), 7.21(\mathrm{~m}$, 2H), $7.45(\mathrm{~s}, 1 \mathrm{H}) ;{ }^{13} \mathrm{C}$ NMR $\left(100 \mathrm{MHz}, \mathrm{DMSO}-\mathrm{d}_{6}\right) \delta 55.4,65.0,110.7,117.9,120.0,127.2,128.3$, 130.9, 147.0, 156.3, 169.0; HRMS (EI) $\mathrm{m} / z$ calcd for $\mathrm{C}_{11} \mathrm{H}_{13} \mathrm{NO}_{3}$ 207.0895, found 207.0891; Anal. Calcd for $\mathrm{C}_{11} \mathrm{H}_{13} \mathrm{NO}_{3} \mathrm{C}, 63.76 ; \mathrm{H}, 6.32 ; \mathrm{N}, 6.76$. Found C, 63.51; H, 6.41; N, 6.53. 


\section{0 ${ }^{1} \mathrm{H}$ NMR Spectra of selected Baylis-Hillman adducts and the addition product 3a}

(Spectra obtained in DMSO- $\mathrm{d}_{6}$ solvent unless otherwise stated)
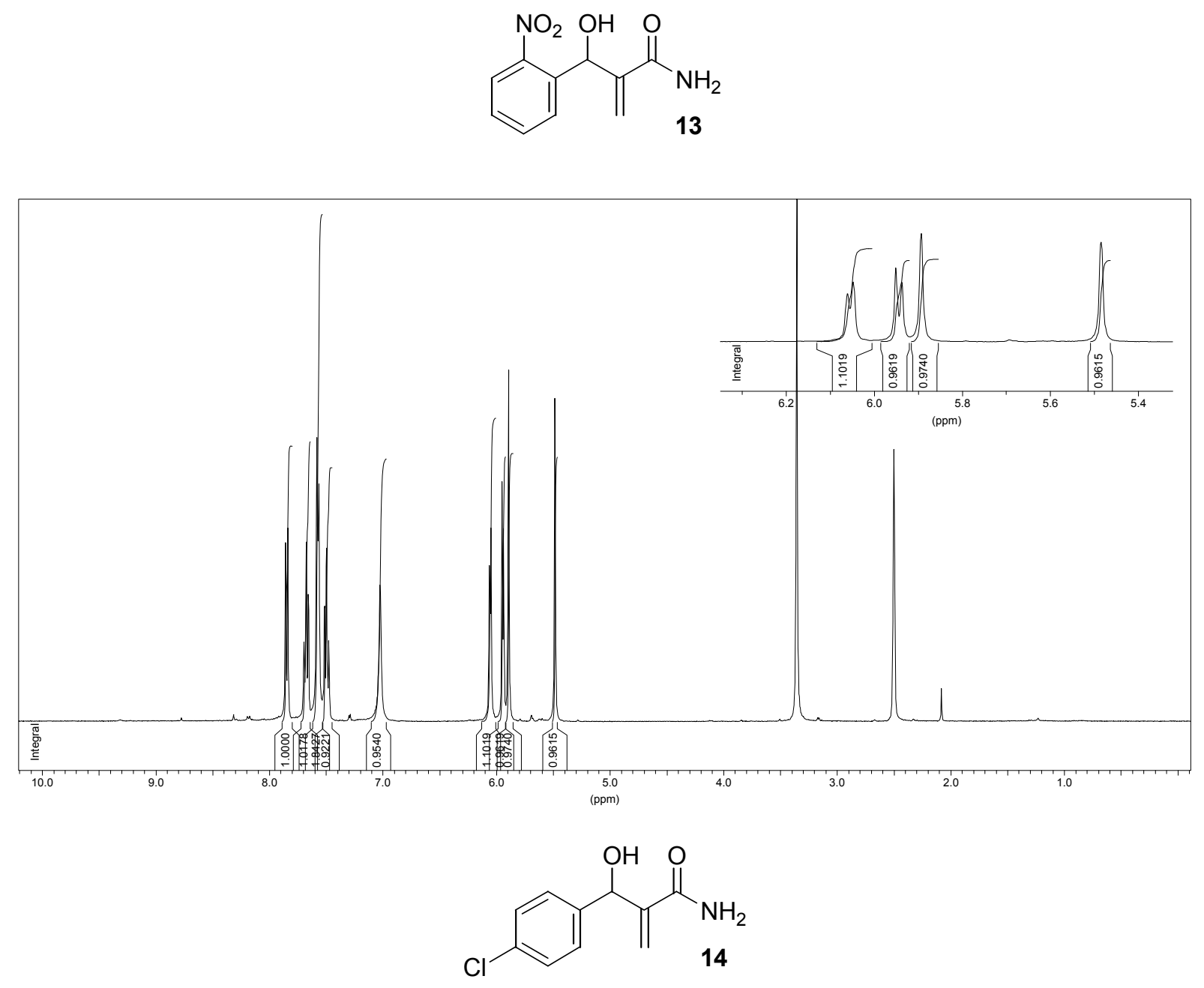

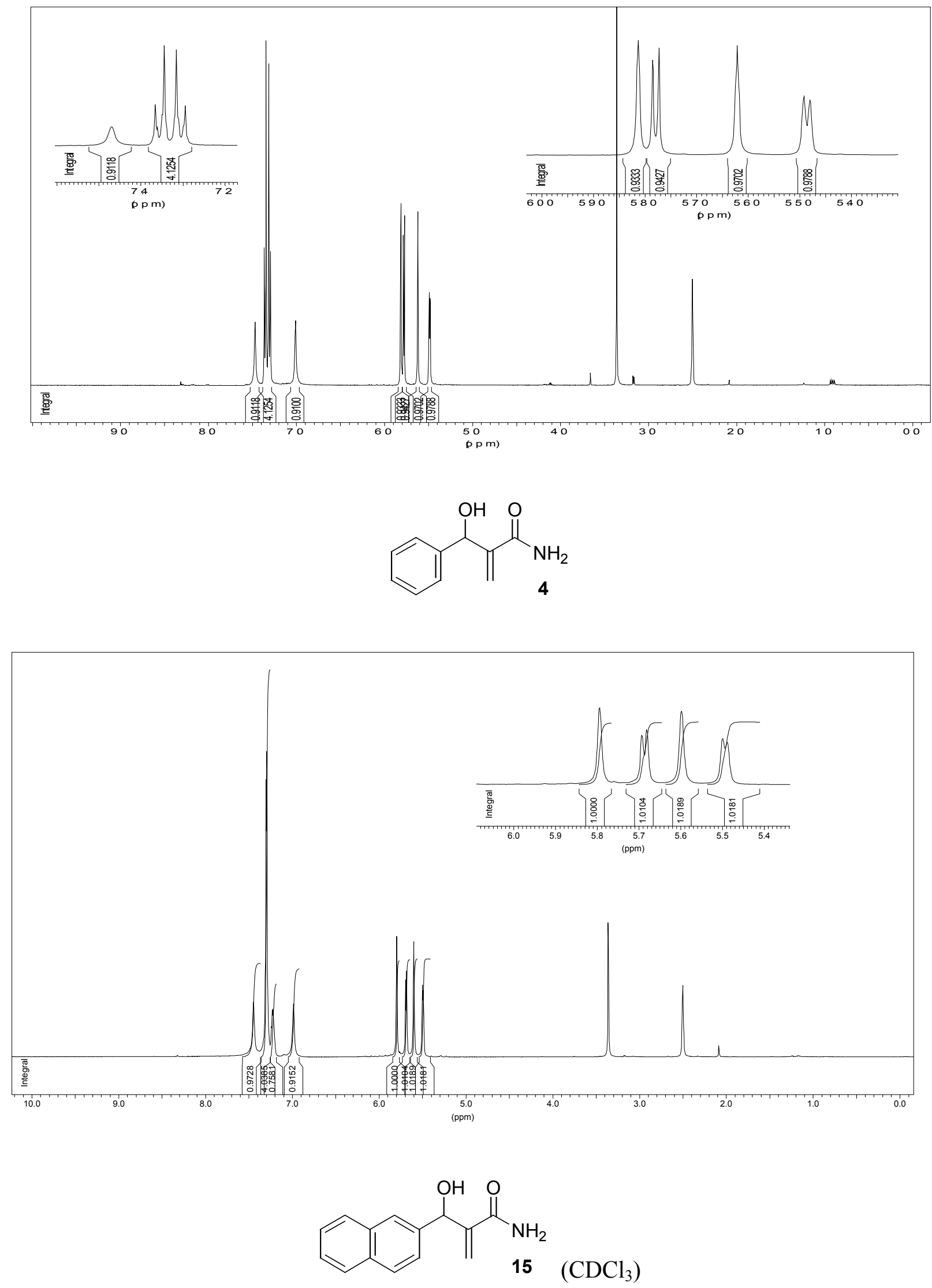

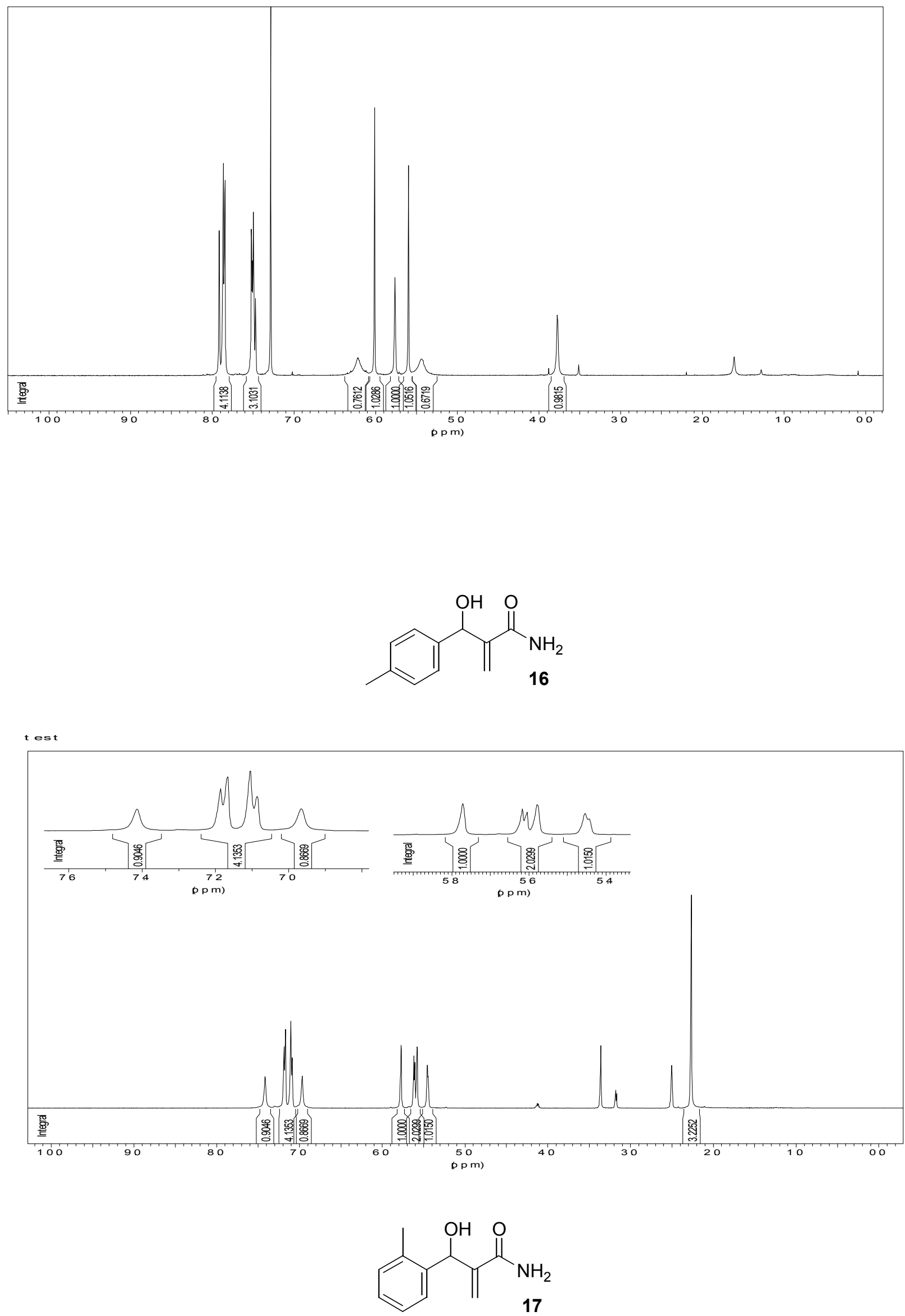

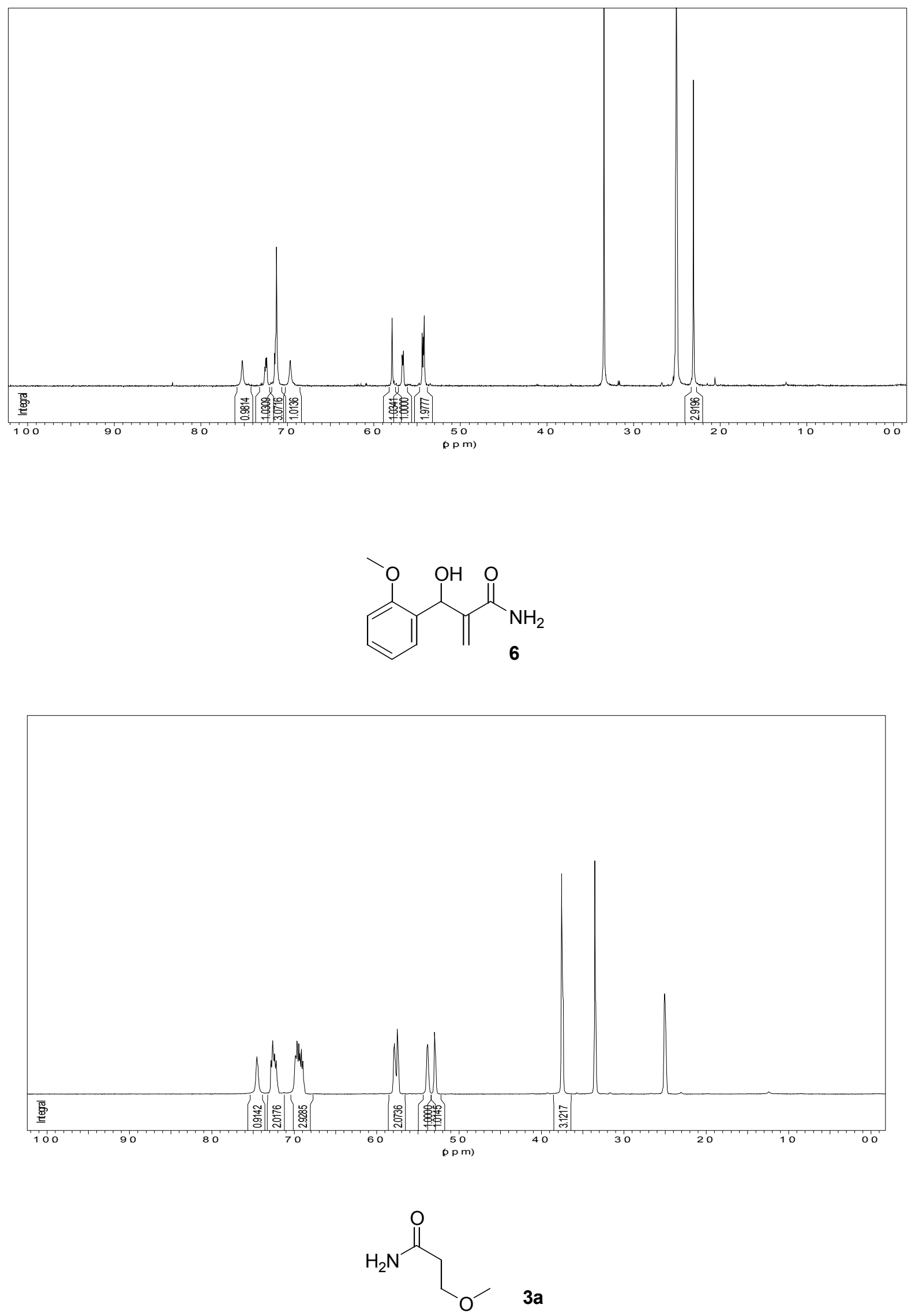

${ }^{1} \mathrm{H},{ }^{13} \mathrm{C}$ and DEPT-135 spectra included ${ }^{4}$ 

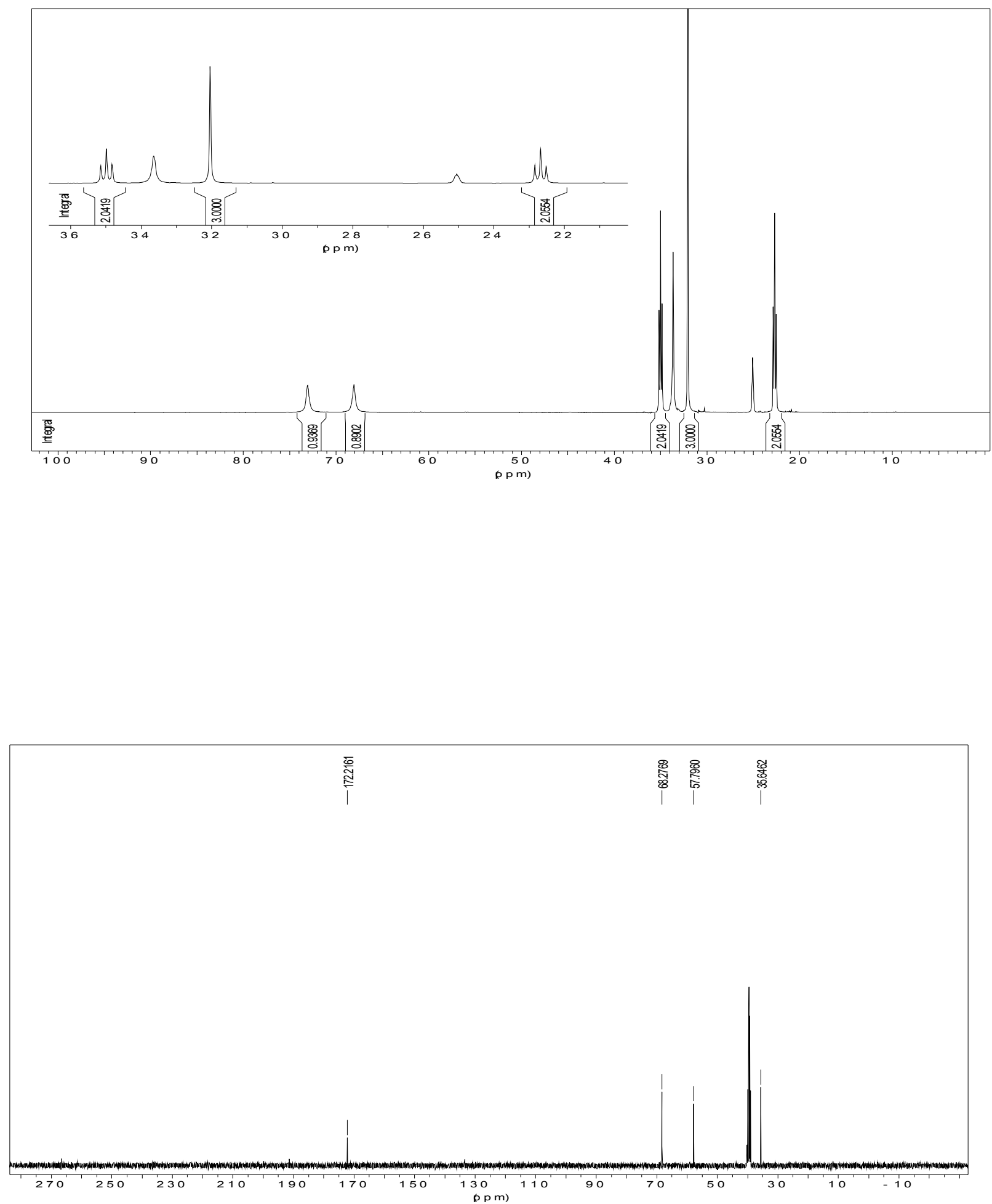


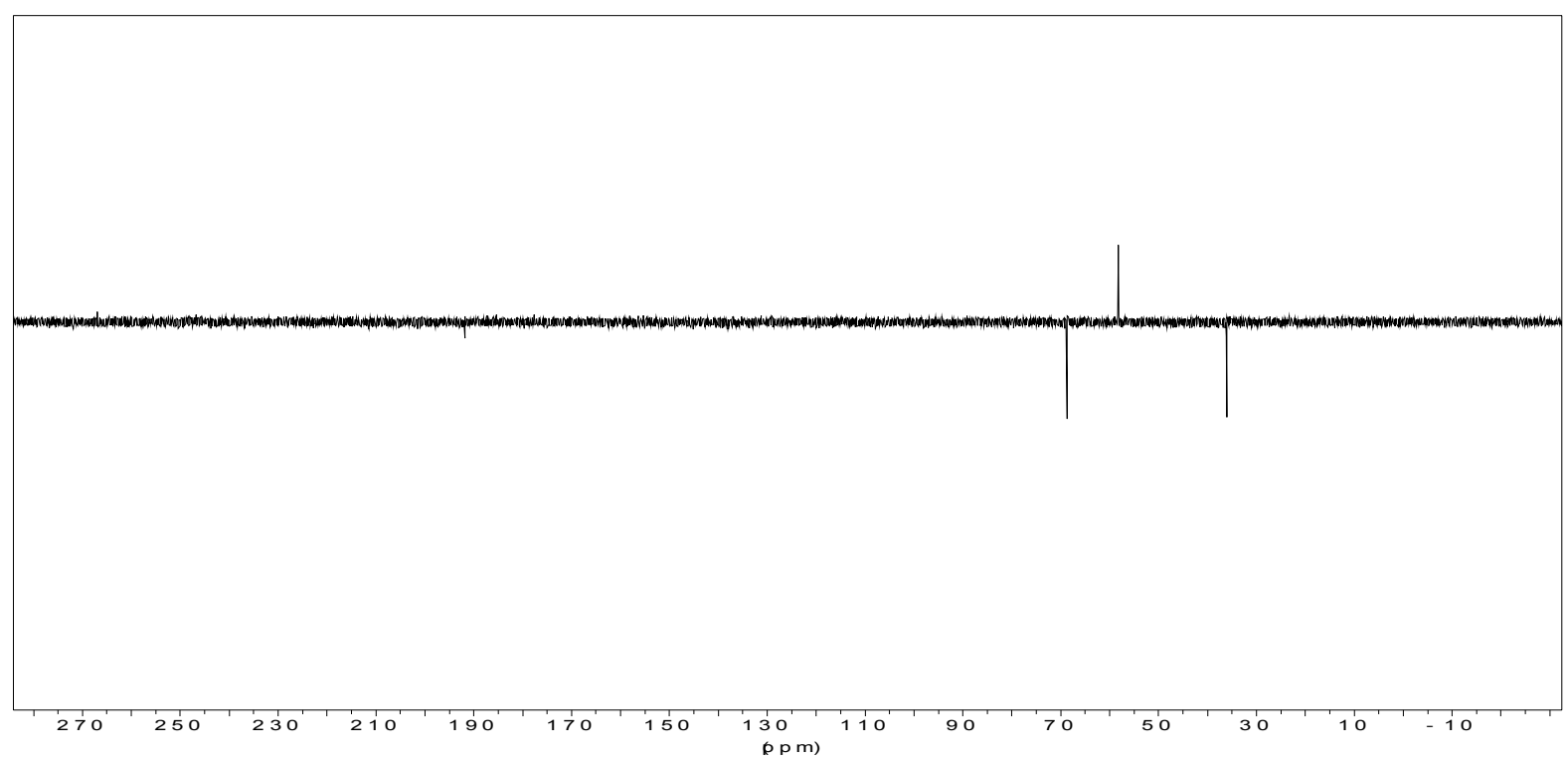

Hydromethoxylation of acrylamide in $\mathrm{MeOH}$ catalysed by DMAP $(\mathrm{t}=1.16 \mathrm{~h}$ - anisole added as an internal standard) showing signals assigned to ammonium intermediate $\mathbf{A}$.

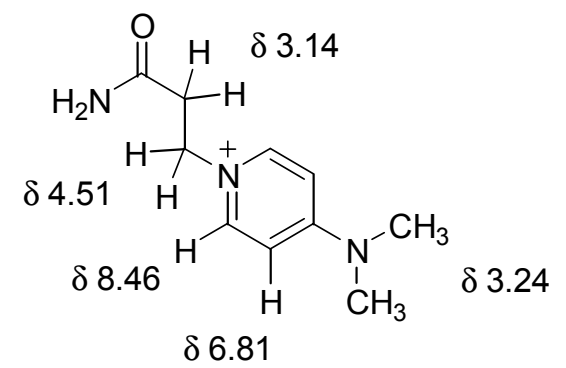




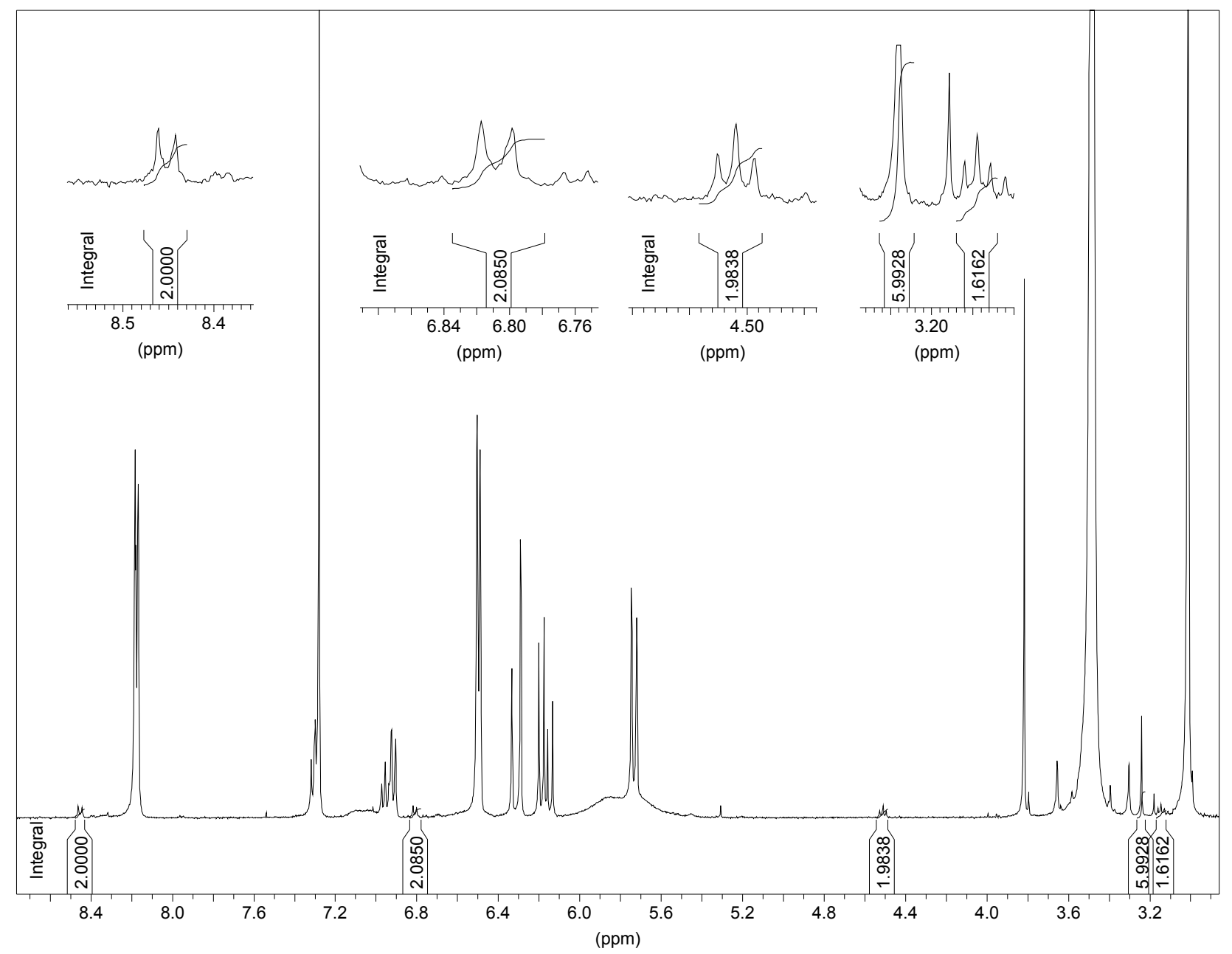

\section{References}

1. We would like to thank Prof. S. Blechert, Technische Universitaet Berlin, for HRMS and elemental analysis data.

2. Yu, C.; Hu, L. J. Org. Chem., 2002, 67, 219.

3. Aggarwal, V. K.; Emme, I.; Fulford, S. Y. J. Org. Chem. 2003, 68, 692. 
4. Dabi, S.; Zilkha, A. Eur. Polym. J. 1977, 13, 545 